Annuaire suisse de politique de développement

13 | 1994

Annuaire Suisse - Tiers Monde 1994

\title{
Les médicaments essentiels
}

\author{
Immita Cornaz
}

\section{OpenEdition}

\section{Journals}

Édition électronique

URL : http://journals.openedition.org/aspd/1060

DOI : $10.4000 /$ aspd. 1060

ISSN : 1663-9669

\section{Éditeur}

Institut de hautes études internationales et du développement

\section{Édition imprimée}

Date de publication : 1 janvier 1994

Pagination : $216-222$

ISSN : 1660-5934

\section{Référence électronique}

Immita Cornaz, «Les médicaments essentiels », Annuaire suisse de politique de développement [En ligne], 13 | 1994, mis en ligne le 19 décembre 2012, consulté le 08 septembre 2020. URL : http:// journals.openedition.org/aspd/1060 ; DOI : https://doi.org/10.4000/aspd.1060 


\title{
Les médicaments essentiels
}

\author{
Immita Cornaz
}

\section{Quelle valeur ont les médicaments?}

Le doute n'est pas possible, les médicaments sont utiles, nécessaires et parfois indispensables pour guérir ou du moins soulager les maux. Pour prévenir certaines maladies aussi: sans vaccin, nous subirions encore en Occident des épidémies de poliomyélite; sans vaccin, jamais la variole n'aurait pu être éradiquée, même si nous ne pouvons guère espérer pouvoir établir de sitôt un autre constat de décès de maladie.

L'usage correct d'un médicament peut faire la différence entre la santé et l'infirmité, voire entre la vie et la mort. Cependant, un médicament mal choisi ou mal utilisé dans le meilleur des cas est inutile; il peut aussi mettre en danger la santé, voire la vie - en fait, il n'est pas rare qu'il le fasse.

Un centre de santé dépourvu de médicaments a bien peu d'efficacité et, de ce fait, voit sa crédibilité se réduire; cependant, un service de santé adéquatement fourni en médicaments mais dont le personnel n'a pas été formé pour les utiliser correctement risque bien d'être de peu d'utilité et de causer plutôt des souffrances. Ce n'est pas vrai, comme certains l'ont affirmé, qu'en l'absence de médecin, le médicament est le moyen le meilleur marché et le plus sûr de protéger la santé. Que ce soit le médecin qui prescrit le médicament, le guérisseur ou l'infirmier, que ce soit le pharmacien ou le marchand ambulant auquel le patient s'adresse pour trouver le remède qui pourra l'aider, sans formation adéquate du prescripteur, sans connaissances suffisantes sur le fonctionnement du corps humain, sur la maladie et sur l'action du remède, tout médicament efficace doit être considéré non comme un bienfait mais comme un puissant instrument potentiellement nocif. Et généralement coûteux. 


\section{Avons-nous les médicaments qu'il nous faut?}

De la dizaine de milliers de produits pharmaceutiques vendus de par le monde ce chiffre est modeste, en réalité il y en a plus - tous n'ont pas l'efficacité qu'on leur prête, certains sont inutiles (sauf comme placebo), d'autres sont plus nocifs qu'utiles. Bon nombre de médicaments constituent cependant un précieux moyen de protection de la santé. D'où l'idée qui a pris forme au début des années 70 de ce que l'OMS et l'Assemblée mondiale de la santé ont convenu d'appeler les "médicaments essentiels". On entend par là un choix de médicaments jugés nécessaires pour prévenir, guérir ou soulager de façon satisfaisante la plupart des affections pouvant être influencées par des médicaments. II est généralement reconnu que quelque 200 ou 300 médicaments (y compris les vaccins) permettent d'atteindre cet objectif. Ces médicaments font partie de toute la gamme des produits pharmaceutiques, des plus simples-au prix de revient modique-aux plus complexes.

Ce concept a commencé par susciter la méfiance dans certains milieux et a rencontré l'opposition de l'industrie qui a cherché à le confiner aux populations pauvres des pays pauvres. Mais l'idée a fait son chemin, et il est très largement reconnu aujourd'hui que le principe des médicaments essentiels n'est pas un concept pour une médecine au rabais ou une médecine pour pays pauvres. C'est à bon escient que l'armée suisse et les hôpitaux de pointe tels les hôpitaux cantonaux appliquent ce principe. En effet, il n'est pas né seulement en réaction à la pléthore de médicaments et à l'utilité discutable de certains d'entre eux. II permet aussi de mieux assurer la qualité des soins: le fait de ne travailler qu'avec un nombre réduit de médicaments sélectionnés à bon escient permet aux prescripteurs de bien les connaître. II convient de souligner dans ce contexte que la désignation des médicaments par leur nom générique est déterminante pour l'utilité du principe des médicaments essentiels. Deuxième avantage (qui compte lui aussi autant pour les pays riches que pour les pays pauvres): en diminuant le gaspillage et les pertes, il permet d'abaisser le coût du traitement et d'assurer une meilleure rentabilité des dépenses.

Pour bien soigner les populations et protéger leur santé, il ne suffit pas de veiller au bon choix dans la surabondance de produits pharmaceutiques et à l'approvisionnement des centres de santé - force est de reconnaître que pour certaines maladies, touchant de larges groupes de population, les médicaments font défaut, tant pour le traitement que pour la prévention. Le SIDA est peut-être l'exemple le mieux connu, mais il est loin d'être unique et, surtout, il est atypique: les instituts universitaires et l'industrie pharmaceutique se sont fortement engagés dans la recherche pour la découverte et le développement de médicaments anti-HIV ou anti-SIDA: le marché potentiel pour de tels médicaments est presque prodigieux. Ces mêmes instituts s'intéressent beaucoup moins aux remèdes contre les maladies qui n'offrent pas de potentialités commerciales. Cela est d'autant plus tragique - le mot n'est pas trop fort - que ce sont les populations pauvres qui ne pourront constituer le marché recherché qui sont aussi les populations les plus exposées aux maladies, y compris les maladies orphelines de remèdes. 
L'OMS, ensemble avec le PNUD (Programme des Nations Unies pour le Développement) et la Banque mondiale, gère le Programme de recherche sur les maladies tropicales ou TDR (voir article de J.-P. Gontard, p. 169) qui a précisément pour but de combler cette lacune, au moins pour six maladies. La DDA, dès le lancement du programme a contribué au financement du programme. Reste à savoir si les priorités du programme correspondent en tous points aux besoins les plus importants et s'il veille suffisamment aux conditions dans lesquelles les populations et les pays les plus touchés pourront utiliser les remèdes mis au point, et surtout si des efforts similaires ne devraient pas être faits pour la prévention et pour le traitement d'autres maladies orphelines.

\section{C'est l'usage rationnel des médicaments qui est déterminant}

L'application du principe des médicaments essentiels, c.à.d. le principe d'un choix correct de médicaments qui devraient être disponibles, signifie que sur le plan national, l'enregistrement des médicaments doit se faire selon des critères qui tiennent compte notamment de l'efficacité des médicaments et des risques inhérents ainsi que des besoins de la population et de la santé publique. Pour les différents niveaux de soins de santé et pour les diverses unités de soins de santé, il devrait y avoir des listes types de médicaments (ou "formularies").

Pour le moins aussi important est l'usage rationnel des médicaments. Cela est vrai autant pour le Nord que pour le Sud. L'usage rationnel des médicaments présuppose, outre le choix correct des médicaments mis à disposition, l'information correcte sur les médicaments, tant pour les autorités chargées de l'enregistrement ou chargées de contrôler l'importation que pour les prescripteurs et pour les consommateurs. Une autre condition est une distribution adéquate des médicaments à l'intérieur du pays afin d'en assurer l'accessibilité pour tous. La qualité des médicaments doit en outre être assurée, ce qui présuppose non seulement une production correcte mais aussi de bonnes conditions de stockage et une réglementation adéquate pour l'enregistrement et le contrôle des médicaments. Un autre aspect important touche à la prescription: qui est compétent pour prescrire (et pour vendre) quels médicaments et quels sont les modes de prescription autorisés (ce qui a trait notamment au problème des prescriptions multiples). Et finalement, on ne donnera jamais trop d'importance à la formation du personnel de santé pour l'usage correcte des médicaments.

L'étendue et la complexité de la formation nécessaire variera bien entendu selon le niveau des services de santé: un "curandero" ou un "village health worker" pourra se suffire de savoir reconnaître les symptômes, de savoir l'effet des quelques remèdes à sa disposition, et de connaître les risques auxquels il doit parer. Ces connaissances sont simples sans doute, mais les cours censés les transmettre sont souvent bâclés ou donnés par un personnel ignorant les réalités du terrain, connaissant mal les rudiments didactiques et ne disposant pas de matériel adéquat. Rarement un tel cours réussit à donner aux hommes et aux femmes à qui seront confiés les remèdes les éléments dont ils auront besoin pour 
bien les administrer aux patients du village ou du quartier. Même les infirmiers responsables des services de santé primaires sont souvent insuffisamment formés ou mal appuyés pour l'usage correct des médicaments. Beaucoup a été fait pour améliorer la formation du personnel soignant - beaucoup reste à faire, dans pratiquement tous les pays, du Sud et du Nord. Et beaucoup reste à faire pour une meilleure formation pharmacologique des médecins, de tous les médecins.

La coopération internationale fait peu, beaucoup trop peu pour mettre les pays en mesure d'assurer l'usage rationnel des médicaments. C'est hautement regrettable.

\section{La politique et les actions de la DDA}

L'engagement de la DDA dans le domaine des médicaments date des années 80 , à part le financement de fournitures de médicaments dans le cadre de l'aide financière et l'envoi ou le financement de l'envoi de médicaments et de vaccins dans le cadre de l'aide humanitaire, dès les années 60 jusqu'à nos jours.

Dès 1983, la DDA s'est engagée avec le Mozambique dans le projet de laboratoire de contrôle de qualité des médicaments: mise en place du laboratoire (lié avec celui du contrôle de l'eau et des aliments réalisé avec le gouvernement italien), et formation, en grande partie en Suisse, du personnel responsable du laboratoire. II peut à première vue paraître surprenant d'équiper un pays pauvre d'un laboratoire somme toute sophistiqué. Mais un tel laboratoire permet de parer aux pertes financières et à l'inefficacité des soins de santé, c.à.d. aux traitements sans effet ou aux effets nocifs, dûs à la part très élevée que représentent, dans les livraisons aux pays pauvres, les médicaments ne correspondant pas aux normes ou dont l'échéance de validité est dépassée: jusqu'à un tiers. Il était d'autant plus justifié d'appuyer le Mozambique dans cet effort que ce pays pratiquait une politique des médicaments basée sur le principe des médicaments essentiels, qu'il avait un système de contrôle des importations, qu'il fournissait un effort remarquable pour doter son système de santé de listes de médicaments requis pour les différents niveaux, qu'il dotait le personnel soignant de listes informatives sur les médicaments et leur utilisation et sur les diverses maladies et leurs traitements médicamenteux, listes adaptées aux niveaux de connaissances du personnel, et qu'enfin, le personnel des services de santé de base était très motivé. La guerre a posé les énormes problèmes au pays que l'on sait, mais la mise en place du laboratoire et la formation du personnel mozambicain ont continué sans relâche, en dépit des retards.

Tôt après le lancement par l'OMS du Programme d'action des médicaments essentiels, en 1981, la Suisse a contribué au financement de ce programme. La décision en a été prise d'un commun accord entre la DDA et l'OFSP (Office fédéral de la santé publique), et les deux offices attachent une grande importance à cette approche commune. Une partie de la première contribution était destinée au développement de modules de formation, projet qui était cofinancé par l'industrie pharmaceutique suisse, par l'intermédiaire d'Interpharma. 
Un peu plus tard, la DDA a aussi cofinancé, à la demande d'Interpharma, un projet d'amélioration du système de stockage des médicaments qu'Interpharma a entrepris au Burundi.

Dans le programme de mise en place et de fonctionnement du système de santé d'Ouéssé au Bénin et dans le programme d'appui au système de santé de Sikasso au Mali, réalisés avec l'aide de la DDA, très tôt l'idée de pharmacies villageoises a été introduite.

Le programme de santé réalisé au Bénin, en collaboration avec la Banque mondiale comporte aussi un important volet relatif à la politique des médicaments. De même, le programme de santé urbaine en Tanzanie, lui aussi réalisé avec la Banque mondiale, porte également sur le système de fourniture et de distribution des médicaments, en accord avec la politique des médicaments essentiels de ce pays.

A Madagascar, la DDA coopère avec le Ministère de la santé pour la réalisation de la politique des médicaments, notamment pour les soins de santé primaires.

Dans l'ensemble du financement de la DDA dans le domaine de la santé, la part des actions dans le domaine des médicaments est certes honorable. Vu cependant l'importance d'une bonne politique des médicaments pour la meilleure protection de la santé, et vu l'impact financier très lourd que peut avoir une mauvaise politique des médicaments, notamment causé par les pertes et le gaspillage et par les suites de traitements inadéquats résultant d'un usage irrationnel de médicaments, l'on peut se demander si la DDA ne devrait pas accroître encore son aide aux pays visant à renforcer leur capacité de réaliser une bonne politique des médicaments.

Parallèlement à l'appui direct aux pays dans le domaine des médicaments, la DDA a aussi cherché à définir sa politique en la matière. Cette définition a d'emblée été entreprise sur un plan interdépartemental, d'un commun accord avec l'OFSP et avec l'OFAEE (Office fédéral des affaires économiques extérieures). En 1984, un document a été mis au point, définissant, à l'intention de l'administration fédérale et des ambassades de Suisse dans les pays en développement, les principes qui guident les actions que le Gouvernement suisse pourra entreprendre dans le cadre de la coopération au développement; il vaut également comme document de référence pour d'autres aspects des relations de la Confédération avec les pays en développement dans le domaine des médicaments. Ce document reconnaît l'importance des médicaments pour la protection de la santé et le bienfondé du principe des médicaments essentiels. II souligne aussiqu'il est hautement souhaitable que les pays en développement aient une politique pharmaceutique cohérente et adaptée au contexte propre à chaque pays, visant à assurer l'accès aux médicaments essentiels à l'ensemble de la population. II poursuit en définissant les conditions qui doivent être remplies pour atteindre ce but. Parmi les principes guidant la politique suisse de coopération au développement en la matière, il est recommandé notamment qu'une attention particulière soit accordée, entre autres, au principe des médicaments essentiels, à l'utilisation du nom générique, à l'adéquation des médicaments et vaccins retenus, à l'information médicale et pharmaceutique, à la qualité des produits, et à la formation en la matière. 
Ce document a été un guide utile pour les actions bilatérales. II a aussi été particulièrement propice sur le plan des relations internationales de la Suisse. Sans cette référence, il aurait été beaucoup plus difficile pour la Suisse de contribuer comme elle l'a fait au débat international, notamment dans le cadre de l'OMS et de I'UNICEF. Ainsi, la délégation suisse à l'Assemblée mondiale de la santé de 1985 a pu intervenir constructivement au débat sur la tenue de la Conférence internationale sur l'usage rationnel des médicaments, demandé par les pays nordiques, en faisant comprendre la nécessité du dialogue entre toutes les parties concernées, ce qui a permis finalement d'obtenir l'accord de l'Assemblée sur la tenue de cette Conférence.

La Conférence a eu lieu en novembre 1985 à Nairobi et elle a constitué une étape décisive dans la prise de conscience internationale de l'importance et des enjeux de la politique des médicaments, politique qui doit faire partie intégrante de la politique de santé de chaque pays. La Conférence a conduit à la définition de la "Stratégie révisée des médicaments", document important d'orientation tant pour les relations internationales que pour les politiques nationales.

Les participants à cette conférence étaient invités par le Directeur général de l'OMS en leur qualité personnelle. Trois Suisses étaient parmi les invités. Un membre de Ciba Geigy, Ernst Vischer, et du côté gouvernemental, le Prof. Beat Roos, Directeur de I'OFSP, et Immita Cornaz de la DDA. Les deux invités gouvernementaux, s'inspirant des principes de la politique suisse, ont élaboré un document de travail sur l'usage rationnel des médicaments. Ce document a été discuté et approuvé avant la Conférence au sein d'un groupe réunissant des représentants en Suisse des divers milieux concernés. Il a eu un impact certain sur les discussions à Nairobi, notamment par l'accent qu'il met sur la responsabilité de chacune des parties concernées. "L'esprit de Nairobi" est marqué aussi par la reconnaissance de la responsabilité partagée. Depuis la Conférence de Nairobi, plus personne ne peut nier cette responsabilité - il n'est pas certain cependant qu'elle soit vraiment assumée par tous.

\section{Pas de développement sans engagement personnel}

Ailleurs dans ce dossier - dans l'aperçu global de la coopération suisse au développement - il est relevé, à juste titre avec une pointe de regret, que les initiatives de réforme ou de modification dans le domaine de la santé souvent sont le fait d'individus isolés. Cela est vrai aussi dans le domaine de la politique des médicaments: les nouvelles voies ont généralement été ouvertes par des personnalités visionnaires ou engagées (ou les deux à la fois), qui se sont presque toujours heurtées à l'inertie sécrétée par la méfiance ou par l'indifférence née de l'ignorance. Dans tous les pays, nous sommes loin de la réalisation de la "Stratégie révisée des médicaments", les arguments purement économiques, épousant la perspective du producteur, sont plus puissants que jamais. Néanmoins, la reconnaissance gagne du terrain que le concept des médicaments essentiels a d'importants avantages - tant pour l'économie que pour la protection de la santé -, qu'il 
est indispensable que chaque pays formule sa politique des médicaments et que la promotion de l'usage rationnel des médicaments reçoive une haute priorité. Cette prise de conscience n'aurait probablement jamais eu lieu sans l'engagement de personnes individuelles - qui, il est vrai, n'ont pas toujours réussi à entraîner les groupes et les institutions - et il est encourageant de noter que c'est la rencontre entre personnalités qui a permis ces démarrages.

La Suisse, terre propice à la production pharmaceutique, semble aussi avoir été un terrain de rencontre de ceux qui ont fomenté la pensée politique et stratégique en matière de médicaments. Pour ne citer que quelques exemples: à Genève, le Dr Halfdan Mahler, alors Directeur général de l'OMS, assisté par l'esprit subtile et habile du Dr Cohen, a lancé la discussion sur les médicaments essentiels et créé le Programme d'action des médicaments essentiels. Le succès, reconnu par tous, de la Conférence de Nairobi sur l'usage rationnel des médicaments, tant redoutée par certains, est dû à l'intelligence mais aussi au courage du Dr Mahler; il a réussi à obtenir l'appui unanime de l'Assemblée mondiale de la santé, à Genève, à la "Stratégie révisée des médicaments". A Lausanne, le professeur Georges Peters a initié ses étudiants à une réflexion critique sur l'usage des médicaments. C'est grâce à un médecin algérien, personnellement lié à la Suisse et fortement engagé dans la problématique des médicaments, que la DDA a sollicité la participation du professeur Peters à son groupe de réflexion sur la santé. Le Dr Helder Martins, premier ministre de la santé du Mozambique et initiateur du système pionnier de soins de santé primaires mozambicain, bien avant la Conférence d'Alma Ata, a reçu une partie de sa formation en Suisse, et c'est lors de son apprentissage professionnel dans l'industrie pharmaceutique suisse qu'il a réuni les éléments qui lui ont permis de concevoir la politique des médicaments de son pays. Sans le respect réciproque entre un autre ancien étudiant de Lausanne, le Dr Pascual Mocumbi, devenu plus tard le deuxième ministre de la santé du Mozambique, et le professeur Georges Peters, peut-être que l'idée de créer un laboratoire de contrôle des médicaments à Maputo ne serait jamais née et c'est cette alliance Mocumbi - Peters qui a fait que d'emblée la DDA s'est intéressée à ce projet somme toute peu ordinaire.

Avec la crise économique et les bouleversements politiques partout dans le monde, les problèmes liés à la réglementaton pharmaceutique ainsi qu'à l'approvisionnement en médicaments et à leur usage se sont aggravés dans presque tous les pays, ce qui a des conséquences parfois dramatiques dans les pays les plus pauvres. Beaucoup reste à faire pour surmonter ces problèmes et pour renforcer la collaboration entre les diverses parties concernées. Plus que jamais il faudra l'intelligence, le courage et l'engagement personnel de ceux qui ont à coeur que toutes les parties concernées puissent assumer la responsabilité qui leur revient. 